\title{
Contribution by males to intraspecific variation of the light dependency of mating in the Drosophila melanogaster species subgroup
}

\author{
Takaomi Sakai $^{1}$, Kunio Isono ${ }^{2}$, Masatoshi Tomaru ${ }^{1}$, \\ and Yuzuru Oguma ${ }^{1, *}$ \\ ${ }^{1}$ Institute of Biological Sciences, University of Tsukuba, Tsukuba, Ibaraki 305, and \\ ${ }^{2}$ Graduate School of Information Sciences, Tohoku University, Sendai 980-77, Japan
}

(Received 2 October 1997, accepted 30 October 1997)

\begin{abstract}
Seven species of the Drosophila melanogaster species subgroup were compared for mating success under different light conditions (light or dark). All species except $D$. melanogaster showed a significantly lower frequency of mating success in the dark than in light in a one-hour mating experiment. Among the six light-dependent species, $D$. sechellia showed the lowest frequency in the dark and $D$. simulans showed the highest in the dark. They were chosen for further intraspecific comparisons using four strains respectively. A substantial difference in the mating frequency in the dark was again found among the four strains in both $D$. sechellia and $D$. simulans, respectively. In these two species reciprocal mating experiments were then performed between the two strains showing the highest and the lowest frequency in the dark. In both species males from the strains with a low mating frequency also showed low scores with the females from the strain that showed a high score. It is therefore shown that males predominantly contribute to the low frequency of mating success in the dark while females appear to make a negligible contribution to the light dependency of mating. The male flies in both $D$. sechellia and $D$. simulans showing high mating frequency did not always show a high locomotor level under light or in the dark. We conclude that the decrease of the mating frequency in the dark is caused by the light-dependent mechanism of male-specific sexual behavior rather than by a mechanism associated with general activity.
\end{abstract}

\section{INTRODUCTION}

Mating of the fruitfly, Drosophila, consists of the sequential elements of characteristic behavior and has been studied by many workers in D. melanogaster and its sibling species (e.g., Bastock and Manning, 1955; Cobb et al., 1985; Welbergen et al., 1987; Cobb et al., 1989). Like many insects a female sex pheromone, which is a main cuticular hydrocarbon, elicits male courtship in these species (Jallon, 1984; Cobb and Jallon, 1990). Males, furthermore, use the female sex pheromone to distinguish their partners (Cobb and Jallon, 1990; Coyne et al., 1994). The sound produced by male wing vibration, the courtship song, is species-specific and plays an important role in sexual excitement for the female as well as in distinguishing male species (Bennet-Clark and Ewing, 1969; von Schilcher, 1976; Cowling and Burnet, 1981; Kyriacou and Hall, 1982). Thus the chemical signals and the auditory signals play active roles to trigger and control courtship in Drosophila (Ewing, 1983;

\footnotetext{
* Corresponding author.
}

Tompkins, 1984; Hall, 1994; Cobb and Ferveur, 1996).

In many species of Drosophila the mating activity is known to be under the control of light. In the dark each species shows a characteristic degree of reduction in the rate of mating success as compared with that in light (Philip et al., 1944; Wallace and Dobzhansky, 1946; Spieth and Hsu, 1950; Narda, 1966; Grossfield, 1966, 1971b; Spieth, 1969; Curtright and Miller, 1979; Oguma et al., 1987). Based on such light dependency of mating in Drosophila, it has been believed that males and females utilize the visual signals mediated by light on mating. In contrast with the roles of the pheromone and the courtship song, it is not yet clear how much the visual information is involved in the mating behavior of males and females. Male visual mutants of $D$. melanogaster show many courtship breaks, extended courtship latency (courtship latency: the time from the initial introduction into a chamber to when male courtship was first observed) and reduced courtship duration (courtship duration: time spent by a male courting a female) (Crossley and Zuill, 1970; Markow and Manning, 1980; Tompkins et al., 1980; Tompkins, 1984). In spite of 
such behavioral abnormalities this species does not show any reduction of mating success in the dark as compared with that in light (Spieth and Hsu, 1950; Rendel, 1951; Manning, 1959; Grossfield, 1966, 1971b). This raises the question of whether the abnormal courtship behavior of males is actually responsible for the low mating success in the dark.

Some Drosophila species have the different degree of light dependency on mating between sibling species (Wallace and Dobzhansky, 1946; Spieth and Hsu, 1950; Manning, 1959; Grossfield, 1971a; Curtright and Miller, 1979). Wallace and Dobzhansky (1946) found that D. subobscura, D. persimilis, and D. pseudoobscura, belonging to the obscura group, show different light dependency of mating. They suggested that such differences may lead to sexual isolation among these species. Grossfield (1971b) interpreted that the different light dependency of mating among sibling species in Drosophila may lead to habitat selection. To acquire evolutionary knowledge on light dependency of mating in Drosophila, the study of behavioral and/or neural mechanism in light dependency of mating and comparison of these differences among sibling species is needed.

Locomotor activity in flies plays an important role in Drosophila mating success. Kyriacou (1981) has concluded that high locomotor activity levels were positively correlated with superiority in male mating performance in $D$. melanogaster. Cobb et al. (1987) have reported that the high locomotor activities in males led to shorter latencies and/or higher levels of courtship in the $D$. melanogaster species subgroup. The locomotor level may be different between light and dark or among species or strains, and thus it may lead to higher levels of courtship, followed by higher levels of copulation.

In this report we compared the mating activity in the dark or light among seven species of the D. melanogaster species subgroup. In $D$. sechellia and D. simulans, comparisons between two light conditions were performed by using a further three strains, indicating a strain specific difference exists in the light dependency of mating. The differences among strains were then analyzed in mating experiments between males and females of inter- and intra-strain combinations under different light conditions to know which sex contributes to the strain specific mating activity and also to the light dependency. In these strains, we studied locomotor activities in light and dark to know whether the strain showing high mating frequency always shows a high locomotor level under light or the dark.

\section{MATERIALS AND METHODS}

Stocks. Strains of the D. melanogaster species subgroup for the mating experiments were D. melanogaster (OregonR), D. simulans (Otsuki, Otsuki; Ogasawara, Ogasawara; Naze, Naze; O-9, Oita), D. mauritiana (G35, Mauritius), D. sechellia (SS51, SS77, and SS78, Plaslin island; SSJ, Cousin islet), D. yakuba (L42, Nairobi), D. teissieri (Bloomington stock center no. 1015) and D. erecta (Ivory coast). All strains except $D$. melanogaster were derived from a single or a few wild-caught females and have since been kept in the laboratory for more than nine years. They were grown on glucose-yeast-cornmeal medium at $25 \pm 0.5^{\circ} \mathrm{C}$ in a $14 \mathrm{~L}$ (7:00-21:00):10 D cycle. For the mating experiments virgin males and females were collected without anesthesia within eight $\mathrm{h}$ after eclosion. Virgin males and females were maintained separately in a vial for 3-6 days until they were used for the experiment.

Mating experiments. Mating experiments were performed between 9:00 and 13:00 at $24.5 \pm 0.5^{\circ} \mathrm{C}$. Fifteen males and ten females were introduced into a vial $(3 \mathrm{~cm}$ diameter $\times 10.5 \mathrm{~cm}$ high) containing food under a dark red photographic safelight (No. 8 Safe Light Grass, HANSA), and were immediately placed in a light-tight box $(30 \mathrm{~cm} \times$ $38 \mathrm{~cm} \times 30 \mathrm{~cm})$ for the dark experiment. The mating frequency in light were performed in a light room. Males and females were allowed to mate for one h. After the experiment female reproductive organs were dissected out and inspected for the presence or absence of sperm. Each of the mating experiments was carried out 8-12 times. Mating frequency was calculated as the percentage of the number of inseminated females over the number of dissected females. The rate of insemination was considered to be an indication for the mating activity.

Measurement of locomotor activity. A single male fly was introduced into a $6 \times 6 \times 0.3 \mathrm{~cm}$ acrylic plastic chamber with an aspirator. A paper ruled into $1 \mathrm{~cm}$ squares was laid under the chamber. After two or twenty min to recover from the disturbance of introduction (2-min and 20-min experiments, respectively), we counted the line numbers which the flies crossed within one min (Connolly, 1967). Thirty males were observed in both the dark and light. To study locomotion in the dark, we observed the flies under the dark red photographic safelight $(20 \mathrm{~W})$ maintained at a distance of approximately $120 \mathrm{~cm}$ from the flies. Since Drosophila do not respond to red light, this condition mimics the effects of total darkness on the flies (Tompkins et al., 1982; McRobert and Tompkins, 1987; Bixler et al., 1992).

Statistical analysis. The chi-square test with Yate's correction was used for comparisons of the mating frequency. When an expected value was less than five, we used the two-tailed Fisher's exact test. The Mann-Whitney $U$ test was used for comparisons of locomotor activity.

\section{RESULTS}

Mating frequency for one $\mathrm{h}$ in the dark or light was investigated in a strain from each of the seven species. Figure 1 shows that $D$. melanogaster attained a high mating fre- 
quency in the dark as well as in light, as reported by others (Spieth and Hsu, 1950; Manning, 1959; Grossfield, 1966). D. simulans, D. sechellia, D. mauritiana, D. yakuba, D. teissieri, and D. erecta each showed a significantly lower mating frequency in the dark than in light (Fig. 1). The results with $D$. simulans, $D$. mauritiana, and $D$. sechellia were similar to data of Manning (1959), Robertson (1983), Cobb et al. (1989) and Cobb and Ferveur (1996). This is the first report that D. yakuba, D. teissieri, and D. erecta showed lower mating frequency in the dark than in light.

Among the six light-dependent species, $D$. simulans and D. sechellia were one of the species showing the least and greatest decrease of mating frequency in the dark, respectively, as compared with the activity in light (Fig. 1). For each of these two species, we added three more strains for further studies. In all the four strains of $D$. sechellia, the mating frequency in the dark were considerably lower than those in light (Table 1). In addition, there were strain differences both in light and dark; in light the strain SS78

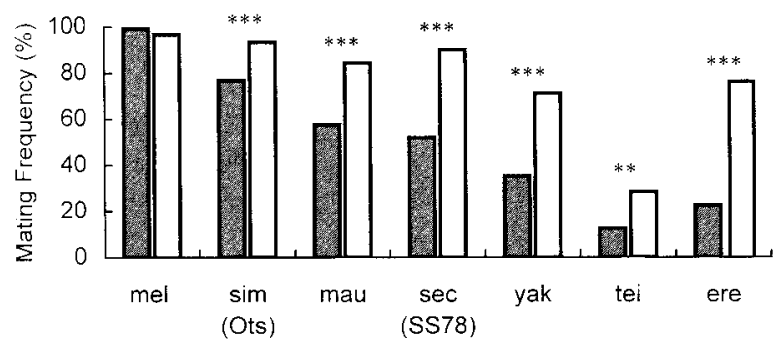

Fig. 1. Mating frequency of the one hour experiment in the dark (solid bars) and light (open bars) of D. melanogaster (mel), D. simulans (sim), D. mauritiana (mau), D. sechellia (sec), D. yakuba (yak), D. teissieri (tei) and D. erecta (ere). Numbers of dissected females were 120 in each strain. **, p $<0.01$; **, $\mathrm{p}<0.001$.

Table 1. Mating frequency (\%) of one hour experiment in light and dark on four strains of D. sechellia and $D$. simulans

\begin{tabular}{lllll}
\hline \hline Species & Strains & Light & Dark & $\chi_{\mathrm{c}}{ }^{2}$ \\
\hline \multirow{2}{*}{ D. sechellia } & SSJ & $80.0^{\mathrm{b}}$ & $27.5^{\mathrm{e}}$ & $64.43^{\mathrm{c}} * * *$ \\
& SS51 & $66.7^{\mathrm{c}}$ & $15.8^{\mathrm{e}}$ & $61.90^{*} * *$ \\
& SS77 & $68.2^{\mathrm{bc}}$ & $20.0^{\mathrm{e}}$ & $52.40 * * *$ \\
& SS78 & $90.0^{\mathrm{a}}$ & $51.7^{\mathrm{d}}$ & $40.84 * * *$ \\
D. simulans & Otsuki & $93.3^{\mathrm{f}}$ & $76.7^{\mathrm{h}}$ & $11.80^{\mathrm{h}} * *$ \\
& Naze & $90.0^{\mathrm{f}}$ & $76.7^{\mathrm{h}}$ & $6.75^{*} *$ \\
& O-9 & $94.2^{\mathrm{f}}$ & $81.7^{\mathrm{h}}$ & $7.69 * *$ \\
& Ogasawara & $92.5^{\mathrm{f}}$ & $92.5^{\mathrm{g}}$ & 0.06 \\
\hline
\end{tabular}

The chi-square test with Yate's correction was used for comparisons between light and dark in each strain. The data of D. sechellia SS78 and D. simulans Otsuki in light and the dark are from Fig. 1. Number of dissected females (n) in all strains were 120 except $D$. sechellia SS77 in light $(\mathrm{n}=110) . \quad \chi_{\mathrm{c}}^{2}$, chisquared value with Yate's correction. **, p < 0.01; ***, p < 0.001 . The values with the same superscript letters are not significantly different by pair-wise comparison of $\chi^{2}$ test with Yate's correction as multiple comparisons in each light condition. showed the highest mating frequency of the four strains of D. sechellia (Table 1). There was no significant difference between other strain combinations except between SS51 and SSJ (Table 1). In the dark, SS78 showed a higher mating frequency than any other strains (Table 1) while the three other strains were not significantly different from each other.

In $D$. simulans, that showed the least decrease of the mating frequency in the dark, there was no significant difference among all the four strains in light (Table 1). In the dark, on the other hand, two strains (Naze and O-9) showed a similar slight decrease of mating frequency as in Otsuki (Table 1). Ogasawara, however, showed a higher mating frequency in the dark than any other strains (Table 1). For Ogasawara, it should also be mentioned that there was no statistical difference in the mating frequency between dark and light as in D. melanogaster (Table 1 and Fig. 1).

Different light dependency within a species observed in both $D$. sechellia and $D$. simulans allowed us to analyze the specific role of sex that may be involved in light-dependent mating. Two strains of D. sechellia, SS78 and SSJ, were chosen for cross-combination of inter-strain mating experiments because they showed a significantly different mating frequencies in the dark (Table 1). In order to know whether the high mating frequency of SS78 pairs is specifically due to males, females or the combination of both sexes, SS78 females were paired with SSJ males and SS78 males, and SSJ females were paired with SSJ males and SS78 males under two light conditions. In light there was no significant difference between the mating frequencies of "SSJ males $\times$ SS78 females" and "SS78 males $\times$ SS78 females" and also between "SS78 males $\times$ SSJ females" and "SS78 males $\times$ SS78 females" (open bars in Fig. 2 ). In the dark, however, SS78 males again showed a high mating frequency with SSJ females as in the intra-strain cross (solid bars in Fig. 2) while SSJ males showed a low value with SS78 females as with SSJ females (solid bars in Fig. 2).

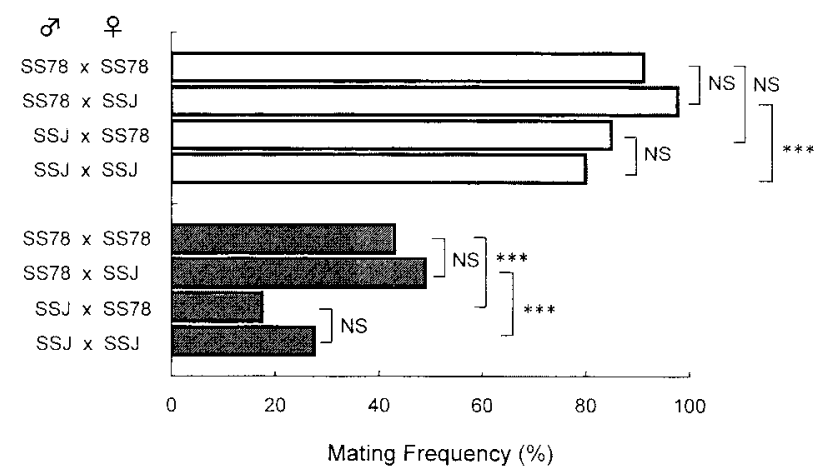

Fig. 2. Mating frequency of the one hour experiment in the dark (solid bars) and light (open bars) of the two strains of $D$. sechellia. The data of SSJ $\times$ SSJ in light and the dark are from Table 1 (SSJ). Numbers of dissected females were 80-120. NS, not significant; $* * *, \mathrm{p}<0.001$. 
Thus the inter-strain mating experiment in $D$. sechellia suggested that males predominantly contribute to the strain difference of mating activity in the dark and females have no or little effect on the activity.

The inter-strain mating experiment was also carried out in D. simulans using two strains: Ogasawara, the light independent strain, and Otsuki, the light-dependent strain (Table 1). As in the case of D. sechellia, it was again shown that the high mating frequency of Ogasawara in the dark can only be attained with the combination of Ogasawara males, not with Ogasawara females (solid bars in Fig. 3). The mating frequency in light, on the other hand, was not significantly different for all the combinations of pairs between Ogasawara and Otsuki (open bars in Fig. 3). It is therefore concluded that in both $D$. sechellia and $D$. simulans the strain difference in mating activity in the dark is predominantly determined by the role of males in each strain.

There is a possibility that locomotion of the flies is influenced by light in a strain-specific manner and that these differences may lead to higher levels of copulations. We studied locomotor activities in light and dark using the two

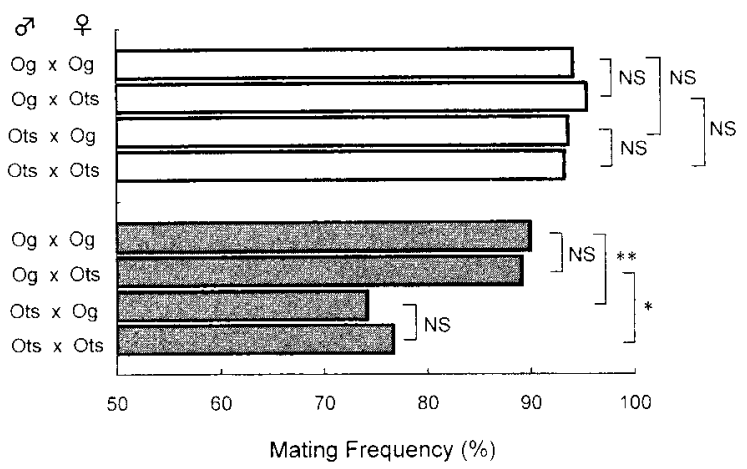

Fig. 3. Mating frequency of the one hour experiment in the dark (solid bars) and light (open bars) of the two strains of D. simulans (Ots and Og are Otsuki and Ogasawara, respectively). The data of Ots $\times$ Ots in light and the dark are from Table 1 (Otsuki). Numbers of dissected females were 100-120. NS, not significant; $*, \mathrm{p}<0.05 ; * *, \mathrm{p}<0.01$. strains of SS78 and SSJ in D. sechellia and two strains of Ogasawara and Otsuki in D. simulans (Table 2). Although there was a slightly significant difference between the 2 min and the 20-min experiments in the dark with D. sechellia SSJ males (Table $2, U=315, \mathrm{p}<0.05$ ), there was no significant difference in comparisons of the other combinations in $D$. sechellia. In both $D$. simulans Otsuki and Ogasawara males, there was a significant difference in locomotor activity at two and twenty min in light (Table 2, in Otsuki males, $U=194$, p < 0. 001; in Ogasawara males, $U=315$, p $<0.05$ ). Otsuki males were more active than Ogasawara males in light at 2-min after introduction (Table $2, U=180$, $\mathrm{p}<0.001)$. There was no significant difference in other comparisons.

\section{DISCUSSION}

We found differences in the light dependency of mating among strains and analyzed the specific role of sex in lightdependent mating. The intraspecific variation in the light dependency of mating was contributed by males, and females had a negligible contribution to the light dependency of mating (Figs. 2 and 3). The locomotor activity in the dark was not lower than that in light in all comparisons (Table 2). It is concluded that the light dependency of mating in Drosophila is not due to the lack of male locomotion in the dark.

Seven species in the $D$. melanogaster species subgroup were investigated for mating frequency in the dark and light. Of these, mating in four strains of $D$. sechellia, three strains of $D$. simulans, and D. mauritiana was light dependent, as had been described by others (Manning, 1959; Robertson, 1983; Cobb et al., 1989; Cobb and Ferveur, 1996). We also confirmed the previous observation that mating in D. melanogaster is light independent (Spieth and Hsu, 1950; Manning, 1959; Robertson, 1983). While Markow (1975), Cobb and Ferveur (1996) and Sakai et al. (1997) had reported that the mating frequency of $D$. melanogaster in the dark was significantly lower than that in light when they

Table 2. Mean locomotor activity levels in light and dark for the male at two or twenty minutes after the introduction to the observation chamber

\begin{tabular}{|c|c|c|c|c|c|c|c|c|c|c|c|}
\hline \multirow{3}{*}{ Species } & \multirow{3}{*}{ Male strains } & \multicolumn{4}{|c|}{ 2-min after introduction } & \multirow{3}{*}{$U$} & \multicolumn{4}{|c|}{ 20-min after introduction } & \multirow{3}{*}{$U$} \\
\hline & & \multicolumn{2}{|c|}{ Light } & \multicolumn{2}{|c|}{ Dark } & & \multicolumn{2}{|c|}{ Light } & \multicolumn{2}{|c|}{ Dark } & \\
\hline & & $\operatorname{mean}^{\dagger}$ & $\mathrm{SE}$ & mean & $\mathrm{SE}$ & & mean & $\mathrm{SE}$ & mean & $\mathrm{SE}$ & \\
\hline \multirow{3}{*}{ D. sechellia } & SSJ & 1.4 & 0.57 & 1.6 & 0.65 & 422.5 & 1.3 & 0.90 & 0 & 0 & 405 \\
\hline & SS78 & 6.5 & 2.49 & 4.8 & 1.55 & 413 & 6.7 & 2.39 & 2.2 & 1.22 & 357 \\
\hline & $U$ & 369 & & 344.5 & & & 326 & & 345 & & \\
\hline \multirow[t]{3}{*}{ D. simulans } & Otsuki & 16.9 & 3.68 & 13.3 & 3.20 & 364 & 3.7 & 1.60 & 6.1 & 2.09 & 402 \\
\hline & Ogasawara & 2.3 & 1.02 & 8.7 & 2.32 & 362 & 0.5 & 0.49 & 4.1 & 1.74 & 357 \\
\hline & $U$ & $180 * * *$ & & 395.5 & & & 372 & & 414 & & \\
\hline
\end{tabular}

The Mann-Whitney $U$ test was used for comparisons between light and dark in each strain of $D$. sechellia and $D$. simulans or between strains in each light condition of two species. $U$, Mann-Whitney's $U$; SE, standard error; ${ }^{\dagger}$, the number of replicates of each experiment is $30 ; * * *, \mathrm{p}<0.001$. 
were mated for a short period, the mating test for one $h$ gave no significant difference (Fig. 1). One of the $D$. simulans strains, Ogasawara, also showed a high mating frequency for one $\mathrm{h}$ in the dark as in light (Table 1). The other three species, D. yakuba, D. teissieri, and D. erecta, which were investigated for the first time in this study, showed significantly lower mating frequency in the dark than in light. It is therefore suggested that the majority of the species belonging to the $D$. melanogaster species subgroup are light-dependent.

Among the four strains of $D$. sechellia, the mating frequencies were different both in the dark and in light (Table 1). There is a tendency that the strain that shows a higher mating frequency in light also shows a higher mating frequency in the dark. Different frequencies of mating success among strains in both light conditions may reflect genetic difference in sexual activities like pheromonal communication and/or general activities like locomotion that ultimately lead to increase in the frequency of mating success. In D. simulans, however, only Ogasawara showed a significantly higher mating frequency in the dark than the other strains while all four strains showed similar mating frequency in light (Table 1). Tables 1 and 2 indicate that male $D$. sechellia SS78 strain flies showing high mating frequency did not always show high locomotor levels and male $D$. simulans Otsuki strain flies showing high locomotor activity did not always show high mating activity under light or dark. The results in Figures 2 and 3 showed that the decrease of mating success in the dark is attributable only to males but not females in D. sechellia and $D$. simulans. Taking these results together, we suggest that the differences in mating frequency among strains in $D$. sechellia and $D$. simulans depend on genetic differences in the light-dependent mechanism of male-specific sexual behavior rather than in the light-dependence of locomotor activity or in the light-independent mechanisms involved in mating activity of the fly.

A similar male-specific light dependency has been well documented in Drosophila eye mutants; in light, white-eyed males of some Drosophila species showed low mating activity (in D. melanogaster, Geer and Green, 1962; Connolly et al., 1969; Burnet and Connoly, 1973; Willmund and Ewing, 1982; Tompkins, 1984: in D. pseudoobscura, Dejianne et al., 1981: in D. auraria and D. biauraria, Isono et al., 1995). Little is known about why the absence of the screening pigments in the eye results in the opposite light dependency in the eye color mutants, but it should be noted that a malespecific visual mechanism that leads to a decrease in the frequency of mating success is involved in both cases. Behavioral changes resulting from visual disturbance have been described by many authors using wild type and mutants of Drosophila as follows: Wild type courting males of $D$. melanogaster show much lower transition probability from orientation to following in the dark than in light (Sakai et al., 1997). D. auraria and D. triauraria males misdi- rect to their females in the attempted copulation in the dark (Oguma et al., 1996). White-eyed males have longer courtship latency (Willmund and Ewing, 1982), and show reduced orientation and wing vibration time than normal males do (Connolly et al., 1969). Male visual mutants of $D$. melanogaster show courtship breaks, extended courtship latency and reduced courtship index (Crossley and Zuill, 1970; Markow and Manning, 1980; Tompkins et al., 1980; Tompkins, 1984). Further behavioral analysis of the members of the $D$. melanogaster species subgroup is needed to identify what behavioral components are associated with the low mating activity in the dark and to examine genetic differences among strains of these species.

We thank T. K. Watanabe and Y. Fuyama for kindly sending us stocks of D. simulans, and D. mauritiana, D. sechellia, D. yakuba, and D. erecta, respectively. We also thank J.-M. Jallon, D. Macer, and $\mathrm{R}$. Weisburd for critically reading the manuscript.

\section{REFERENCES}

Bastock, M., and Manning, A. (1955) The courtship of Drosophila melanogaster. Behaviour 8, 85-111.

Bennet-Clark, H. C., and Ewing, A. W. (1969) Pulse interval as a critical parameter in the courtship song of Drosophila melanogaster. Anim. Behav. 17, 755-759.

Burnet, B., and Connolly, K. (1973) The visual component in the courtship of Drosophila melanogaster. Experientia 29, 488489.

Bixler, A., Jenkins, J.-B., Tompkins, L., and McRobert, S.-P. (1992) Identification of acoustic stimuli that mediate sexual behavior in Drosophila busckii (Diptera: Drosophilidae). J. Insect Behav. 5, 469-478.

Cobb, M., Connolly, K., and Burnet, B. (1985) Courtship behaviour in the melanogaster species sub-group of Drosophila. Behaviour 95, 203-231.

Cobb, M., Connolly, K., and Burnet, B. (1987) The relationship between locomotor activity and courtship in the melanogaster species sub-group of Drosophila. Anim. Behav. 35, 705-713.

Cobb, M., Burnet, B., Blizard, R., and Jallon, J. -M. (1989) Courtship in Drosophila sechellia: Its structure, functional aspects, and relationship to those of other members of the Drosophila melanogaster species sub-group. J. Insect Behav. 2, 63-89.

Cobb, M., and Jallon, J.-M. (1990) Pheromones, mate recognition and courtship stimulation in the Drosophila melanogaster species subgroup. Anim. Behav. 39, 1058-1067.

Cobb, M., and Ferveur, J.-F. (1996) Evolution and genetic control of mate recognition and stimulation in Drosophila. Behav. Proc. 35, 35-54.

Connolly, K. (1967) Locomotor activity in Drosophila III. A distinction between activity and reactivity. Anim. Behav. 15, 149152.

Connolly, K., Burnet, B., and Sewell, D. (1969) Selective mating and eye pigmentation: an analysis of the visual component in the courtship behavior of Drosophila melanogaster. Evolution 23, 548-559.

Cowling, D. E., and Burnet, B. (1981) Courtship songs and genetic control of their acoustic characteristic in sibling species of the Drosophila melanogaster subgroup. Anim. Behav. 29, 924935.

Coyne, J. A., Crittenden, A. P., and Mah, K. (1994) Genetics of a pheromonal difference contributing to reproductive isolation in Drosophila. Science 265, 1461-1464. 
Crossley, S., and Zuill, E. (1970) Courtship behaviour of some Drosophila melanogaster mutants. Nature 225, 1064-1065.

Curtright, R. D., and Miller, D. D. (1979) Light-dependent mating in the Drosophila affinis subgroup. Anim. Behav. 27, 313314.

Dejianne, D., Hotchkiss, A. P., and Grossfield, J. (1981) Light dependent mating inhibition in the white-eye mutant of Drosophila pseudoobscura. Experientia 37, 465-466.

Ewing, A. W. (1983) Functional aspects of Drosophila courtship. Biol. Rev. 58, 275-292.

Geer, B. W., and Green, M. M. (1962) Genotype, phenotype and mating behavior of Drosophila melanogaster. Am. Nat. 96, $175-181$.

Grossfield, J. (1966) The influence of light on the mating behavior of Drosophila. . Univ. Tex. Publ. 6615, 147-176.

Grossfield, J. (1971a) Behavioral differentiation of three races of Drosophila auraria. J. Hered. 62, 117-118.

Grossfield, J. (1971b) Geographic distribution and light-dependent behavior in Drosophila. Proc. Natl. Acad. Sci. USA 68, 26692673.

Hall, J. C. (1994) The mating of a fly. Science 264, 1702-1714.

Isono, K., Lazarova, G., Sakai, T., Tomaru, M., Matsubayashi, H., and Oguma, Y. (1995) Excitatory and inhibitory control of mating activity in Drosophila auraria and biauraria by light. Naturwissenschaften 82, 241-242.

Jallon, J. M. (1984) A few chemical words exchange by Drosophila during courtship and mating. Behav. Genet. 14, 441-478.

Kyriacou, C. P. (1981) The relationship between locomotor activity and sexual behaviour in ebony strains of Drosophila melanogaster. Anim. Behav. 29, 462-471.

Kyriacou, C. P., and Hall, J. C. (1982) The function of courtship song rhythms in Drosophila. Anim. Behav. 30, 794-801.

Manning, A. (1959) The sexual behaviour of two sibling Drosophila species. Behaviour 15, 123-145.

Markow, T. A., and Manning, M. (1980) Mating success of photoreceptor mutants of Drosophila melanogaster. Behav. Neural Biol. 29, 276-280.

Markow, T. A. (1975) Effect of light on egg-laying rate and mating speed in phototactic strains of Drosophila. Nature 258, 712714.

McRobert, S. P., and Tompkins, L. (1987) The effect of light on the sexual behavior of Drosophila affinis. Behav. Neural Biol.47, 151-157.

Narda, R. D. (1966) Analysis of the stimuli involved in courtship and mating in D. malerkotliana (Sophophora, Drosophila). Anim. Behav. 14, 378-383.

Oguma, Y., Kurokawa, H., Akai, S. M., Tamaki, H., and Kajita, J.
(1987) Interspecific differences in some courtship behavioral properties among the four species belonging to the Drosophila auraria complex. Zool. Sci. 4, 889-896.

Oguma, Y., Jallon, J.-M, Tomaru, M., and Matsubayashi, H. (1996) Courtship behavior and sexual isolation between Drosophila auraria and D. triauraria in darkness and light. J. Evol. Biol. 9, 803-815.

Philip, D. U., Redel, J. M., Spurway, H., and Haldane, J. B. S. (1944) Genetics and karyology of Drosophila subobscura. Nature 154, 260-262.

Rendel, J. M. (1951) Mating of ebony vestigial and wild type Drosophila melanogaster in light and dark. Evolution 5, 226-230.

Robertson, H. M. (1983) Mating behavior and the evolution of Drosophila mauritiana. Evolution 37, 1283-1293.

Sakai, T., Isono, K., Tomaru, M., and Oguma, Y. (1997) Light-affected male following beharior is involved in light-dependent mating in Drosophila melanogaster. Genes Genet. Syst. 72, $275-281$.

Spieth, H. T., and Hsu, T. (1950) The influence of light on the mating behavior of seven species of the Drosophila melanogaster species group. Evolution. 4, 316-325.

Spieth, H. T. (1969) Courtship and mating behavior of the Drosophila nasuta subgroup of species. Univ. Tex. Publ. 6918, 255270.

Tompkins, L., Hall, J. C., and Hall, L. M. (1980) Courtship-stimulating volatile compounds from normal and mutant Drosophila. J. Insect Physiol. 26, 689-697.

Tompkins, L., Gross, A. C., Hall, J. C., Gailey, D. A., and Siegel, R. W. (1982) The role of female movement in the sexual behavior of Drosophila melanogaster. Behav. Genet. 12, 295-307.

Tompkins, L. (1984) Genetic analysis of sex appeal in Drosophila. Behav. Genet. 14, 411-440.

von Schilcher, F. (1976) The function of pulse song and sine song in the courtship of Drosophila melanogaster. Anim. Behav. 24, 622-625.

Wallace, B., and Dobzhansky, T. (1946) Experiments on sexual isolation in Drosophila. VIII. Influence of light on the mating behavior of Drosophila subobscura, Drosophila persimilis and Drosophila pseudoobscura. Proc. Natl. Acad. Sci. USA 32, 226-234.

Welbergen, P., van Dijken, F. R., and Scharloo, W. (1987) Collation of the courtship behaviour of the sympatric species Drosophila melanogaster and Drosophila simulans. Behaviour 101,253274.

Willmund, R., and Ewing, A. (1982) Visual signals in the courtship of Drosophila melanogaster. Anim. Behav. 30, 209-215. 